\title{
La Chirurgie Foraine : Une Solution Aux Problemes D'acces Aux Soins Chirurgicaux Des Populations Rurales
}

\author{
Boyodi KatangaTchangai \\ Faculté des Sciences de la Santé /Université de Lomé, Togo \\ Fousseni Alassani \\ Service de chirurgie viscérale / CHU Sylvanus Olympio \\ Faculté des Sciences de la Santé/Université de Lomé, Togo \\ Kossigan Ayi Amavi \\ Clinique MedicoChirurgicale /CHU Sylvanus Olympio, Togo \\ Komlan Attipou \\ service de chirurgie viscérale / CHU Sylvanus Olympio \\ Faculté des Sciences de la Santé/Université de Lomé, Togo
}

doi: 10.19044/esj.2016.v12n36p174 URL:http://dx.doi.org/10.19044/esj.2016.v12n36p174

\begin{abstract}
Assessment of the quality of care in surgical outreach programs is necessary to improve their reliability.

Purpose: The purpose of this study is to evaluate the clinical results of a surgical outreach program involving large numbers of patients.

Material and Method: Retrospective study of clinical data and outcomes of patients operated on within a surgical outreach held in Togo's Central Region was carried out. Data was collected from patient's files with a follow-up, of 2 years.

Results: Two hundred and seventy seven patients (277) underwent 304 surgical procedures within the surgical outreach. The conditions were essentially made of hernias of the abdominal wall (89\%). Cases of hydroceles, endemic goiter and uterine myo-fibroids were also managed. Spinal anesthesia was the most common procedure of anesthesia (75\%). Day case surgery involved 165 patients $(59.5 \%)$. A post operative morbidity was observed in 31 patients (11\%) essentially made of hematoma 28 cases $(9.2 \%)$ and parietal abscesses 20 cases $(6.5 \%)$. There were no postoperative deaths.

Conclusion: outcomes in this study encourage surgical outreach programs as reliable means to address surgery accessibility issues of rural populations in low incomes countries.
\end{abstract}


Keywords: Surgery, outreach, hernia, hydrocele.

\section{Resume}

L'évaluation de la qualité des soins dans les programmes de chirurgie foraine est nécessaire pour garantir leur efficacité.

But: Le but de cette étude est d'évaluer les résultats cliniques d'une mission chirurgicale foraine impliquant un grand nombre de patients.

Méthode: Il s'est agi d'une étude rétrospective des données cliniques d'une mission foraine de soins chirurgicaux de masse effectuée dans la région centrale du Togo. Les données analysées ont été collectées pendant la phase active de la mission et un suivi post opératoire de 2 ans.

Résultats: Deux cent soixante dix sept patients (277) ont bénéficié de 304 procédures chirurgicales dans le cadre de la mission. Les affections opérées ont été essentiellement des hernies de la paroi abdominale (89\%). Des cas d'hydrocèles de la vaginale testiculaire, de goitre endémique et de myofibrome utérins ont été également pris en charge. Le mode d'anesthésie le plus fréquent a été la rachianesthésie, réalisée dans $75 \%$ des cas. La prise en charge ambulatoire a concerné 165 patients $(59,5 \%)$. Une morbidité post opératoire a été observée chez 31 patients $(11 \%)$. Elle était essentiellement faite d'hématomes 28 cas $(9,2 \%)$ et de suppurations pariétales 20 cas $(6,5 \%)$. La mortalité post opératoire a été nulle.

Conclusion: Les résultats de cette étude permettent d'envisager la chirurgie foraine comme une réponse efficace à la problématique d'accessibilité aux soins chirurgicaux en milieu rural.

Mots clés: Chirurgie foraine, hernie, hydrocele.

\section{Introduction}

La réalisation d'actes chirurgicaux nécessite une infrastructure et du personnel adaptés aux exigences sanitaires en matière de soins invasifs. La distribution homogène de ces ressources sur le territoire est un véritable défi dans les pays à ressources limitées (Kushner 2010). Par ailleurs, les populations rurales présentent des difficultés majeures d'accès aux soins chirurgicaux du fait de leur coût relativement élevé. Des études pilotes ont déjà montré qu'il était possible pour résoudre ces problèmes, de proposer à ces populations un programme de chirurgie foraine (Thomas 2009, Aderounmu 2008). Pour amortir les coûts, ces programmes prennent la forme d'interventions chirurgicales de masses qui peuvent susciter un scepticisme raisonnable sur la qualité des soins. Nous rapportons dans ce travail l'expérience d'un projet de soins sous la forme d'une mission chirurgicale foraine de masse en milieu rural. Le but est d'évaluer les résultats cliniques 
obtenus en termes de morbidité, de mortalité, d'efficacité et de donner ainsi des arguments justifiant ces formes d'actions.

\section{Patients et Méthode}

Il s'agit d'une étude rétrospective, d'une cohorte de patients opérés dans le cadre d'un projet de soins chirurgicaux itinérants réalisé du10 au 30 novembre 2013 dans la Région Centrale du Togo. L’objectif de ce projet effectué dans un cadre humanitaire par une ONG locale Togolaise était de rendre accessible aux populations rurales, des soins chirurgicaux. Le projet de soin a été réalisé dans une région de 549179 habitants avec une superficie de $13182 \mathrm{~km} 2$ qui disposait de deux hôpitaux pourvus chacun d'un chirurgien et d'un bloc opératoire soit un ratio de 1 chirurgien pour 274589 habitants. Dans le cadre de la mission, une équipe de 4 chirurgiens généralistes locaux et de 6 infirmiers anesthésistes volontaires a été constituée. Le recrutement des patients a été effectué sur la base de consultations itinérantes gratuites dans toute la région. Les affections ciblées relevaient de la chirurgie générale. Les patients pour lesquels une indication opératoire était proposée réalisaient systématiquement un bilan préopératoire sommaire (numération formule sanguine, urée, glycémie, créatinémie, groupage sanguin) et une consultation pré-anesthésique. Les patients retenus ont été regroupés et opérés dans les 2 hôpitaux sur une période de 5 jours. Les patients jeunes, opérés d'une affection de paroi, sans facteurs de morbidité, résidant dans une localité facilement accessible étaient pris en charge de façon ambulatoire ; les autres, en fonction des suites opératoires immédiates. Dans tous les cas, les patients étaient adressés dans la structure de soins périphérique la plus proche de leur habitation avec des consignes de soins post opératoires. Les patients ont été de nouveau regroupés pour une consultation de contrôle par un chirurgien de la mission 2 ans après les interventions chirurgicales. La collecte des données en vue de l'étude a été effectuée de façon prospective pendant le déroulement de la mission chirurgicale puis rétrospectivement à l'occasion du regroupement des patients 2 ans après la phase active du projet de soins. Les carnets de suivi dans les formations sanitaires périphériques ont également servi à la collecte. Les données démographiques, les indications thérapeutiques et les suites opératoires ont été recueillies sur fiche d'enquête puis traitées et analysées à l'aide du logiciel SPSS.

\section{Résultats}

Deux cent soixante dix sept (277) patients ont été opérés pendant 5 jours dans le cadre du projet de soins forains. L'âge des patients opérés a varié de 3 à 87 ans; la moyenne d'âge était de 37 ans. Le sex ratio était de 5,7 en faveur des hommes. Les affections opérées ont été les hernies de la paroi abdominale antérieure (275 patients), l'hydrocèle de la vaginale testiculaire 
(17 patients), le goitre endémique ( 7 patients) et le léiomyofibrome utérin (5 patients).

Le mode d'anesthésie a été en priorité loco régional avec $75 \%$ des procédures réalisées sous rachi-anesthésie (Tableau I).

Tableau I : Répartition des techniques d'anesthésie

$$
\text { Effectif Pourcentage }
$$

\begin{tabular}{lcc}
\hline Anesthésie locale & 30 & $10 \%$ \\
Bloc ilio inguinal & 13 & \\
Bloc spermatique 17 & \\
Rachianesthésie & 208 & $75 \%$ \\
Anesthésie générale & 39 & $15 \%$ \\
\hline
\end{tabular}

Tableau II : Répartition des interventions réalisées

Effectif Pourcentage

\begin{tabular}{cccc} 
Cure herniaire & 275 & \multicolumn{2}{c}{$89 \%$} \\
Hernie de l'aine & 256 & \multicolumn{2}{c}{$82 \%$} \\
Hernie ombilicale & 19 & \multicolumn{2}{c}{$6 \%$} \\
Cure d'hydrocèle & 17 & \multicolumn{2}{c}{$0,06 \%$} \\
Myomectomie & 2 & \multicolumn{2}{c}{$0,09 \%$} \\
Hystérectomie & 3 & $0,03 \%$ \\
Thyroïdectomie subtotale & 7 & \multicolumn{2}{c}{0}
\end{tabular}

Trois cent quatre (304) gestes opératoires ont été réalisés (Tableau II).En ce qui concerne les hernies de l'aine, la technique de Bassini, a été effectuée dans 216 cas $(84,3 \%)$. La technique de Mac Vay a été réalisée dans 23 cas $(8,3 \%)$, la technique de Shouldice effectuée dans 14 cas $(5,4 \%)$, la herniotomie simple dans 3 cas (1\%). La technique en cas d'hydrocèle a été exclusivement l'excision de la vaginale.

L'antibioprophylaxie pré opératoire a été systématique avec 2 grammes d'ampicilline par voie intraveineuse avant l'incision.

Une morbidité post opératoire a été notée chez 31 patients (11\%). Elle était faite essentiellement d'hématomes et de suppuration de la plaie opératoire (tableau III). Un cas de plaie vésicale et un volumineux hématome post opératoire compliquant dans les deux cas une cure herniaire ont nécessité une ré-intervention. La mortalité post opératoire était nulle. La prise en charge ambulatoire a concerné 165 patients $(59,5 \%)$ parmi lesquels $6(3 \%)$ ont nécessité une réadmission. Chez les autres patients, la durée moyenne d'hospitalisation était de 2,4 jours (extrême de 1 et 9 jours). Une récidive herniaire a été observée essentiellement après la cure d'une hernie de l'aine chez 12 patients $(4,6 \%)$ dans un délai de 11 à 24 mois. Les suites opératoires 
étaient considérées comme simples au terme de la période de suivi chez le reste des patients.

Tableau III : morbidité post opératoire

Effectif

Pourcentage

\begin{tabular}{|c|c|c|c|}
\hline Hématome post opératoire & $289,2 \%$ & & \\
\hline Suppuration pariétale & 20 & $6,5 \%$ & \\
\hline Hydrocèle post opératoire 7 & & $2,3 \%$ & \\
\hline Rétention aigue d'urine & 3 & & $0,9 \%$ \\
\hline Atrophie testiculaire & & & \\
\hline Céphalées post-rachianesthésie & e 5 & & $1,6 \%$ \\
\hline Plaie de la vessie 1 & $0,3 \%$ & & \\
\hline
\end{tabular}

\section{Discussion}

La chirurgie itinérante ou foraine est une pratique inspirée de la médecine humanitaire et de catastrophe dans laquelle des infrastructures médicales mobiles sont déployées au plus près des sinistrés (Ginzburg 2010). L'objectif est de proposer une chirurgie de proximité aux populations présentant des difficultés d'accès aux soins (Thomas 2009, Aderounmu 2008). L'aspect forain des soins, et le nombre important des patients opérés impliquent des conditions particulières de travail qui peuvent impacter la qualité des gestes effectués. Comme dans la chirurgie humanitaire, les indications, les modalités d'anesthésie et même les techniques chirurgicales doivent être adaptées au contexte pour limiter la morbidité et la mortalité opératoire.

La hernie de l'aine a été la plus fréquente des affections opérées soit $82 \%$ de l'ensemble des interventions. Ces affections représentent l'essentiel de la pratique chirurgicale non urgente en milieu rural africain (Harouna, 2000). Leur prise en charge, en considérant le rapport coût et service médical rendu, est parfaitement adapté au concept de soins forains. L'hydrocèle de la vaginale testiculaire, affection fréquente en milieu tropical (Programme Mondial pour l'Elimination de la Filariose Lymphatique, 2002) était sous représentée dans notre recrutement $(5 \%)$ en lien avec l'existence de programmes spécifiques existant dans la région. Il existe une diversité d'affections en milieu rural qui ont été peu traitées au cours de la mission. Leur prise en charge dans le cadre d'une mission itinérante doit être mesurée en tenant compte de la capacité de l'équipe soignante locale à gérer les suites opératoires. Elles posent également, le problème des explorations paracliniques que l'on sait difficile à obtenir pour des raisons financières et de disponibilité en milieu rural. 
Les techniques d'anesthésie ont été essentiellement locorégionales. Cela correspond à la distribution des affections traitées (essentiellement des hernies), à la nécessité de s'adapter aux faibles capacités d'accueil des hôpitaux ruraux et à leur plateau technique limité. L'idéal, en situation foraine, aurait été de privilégier les procédures d'anesthésie locale qui sont validées et largement recommandées en matière de hernies de l'aine et d'hydrocèles (Programme Mondial pour 1'Elimination de la Filariose Lymphatique2002, Pélissier 2000).

Les techniques de cure dites sous tension ont été utilisées dans toutes les cures de hernie. Les méthodes prothétiques, indépendamment de leurs excellents résultats ont un coût qui en limite l'utilisation à l'heure actuelle en milieu africain et rural (Pallas 2000). Dans la prise en charge des hernies de l'aine, la technique de Bassini a été la plus réalisée ; ce qui correspond à la réalité de la pratique chirurgicale en Afrique subsaharienne où cette méthode connait une large utilisation (Harouna 2000, Koumaré 1991). Le taux de récidive obtenu dans cette mission (4\%) est comparable aux données de la littérature concernant les méthodes dites sous tension, qui varient de 0 à $30 \%$ avec une moyenne de 8,6\% pour la technique de Bassini (Pélissier 2000, Hay 1995).

La technique utilisée pour la cure d'hydrocèle a été essentiellement l'excision de la vaginale testiculaire. La préférence pour cette technique est dictée par l'épidémiologie des hydrocèles en milieu tropical qui sont en majorité d'origine micro-filarienne (Programme Mondial pour l'Elimination de la Filariose Lymphatique 2002). Le faible taux de récidive dans cette technique est à mettre en balance avec le risque accru d'hématome de la bourse que l'on peut prévenir par l'hémostase correcte de la collerette vaginale résiduelle.

La morbidité a été dominée par les hématomes (8\%), un fait lié aux caractéristiques des hydrocèles et des hernies de l'aine en milieu rural africain. Leur volume est en effet significativement plus élevé que dans les séries occidentales (Sanders 2008). La morbidité infectieuse, paramètre important de la qualité des soins chirurgicaux venait en deuxième position. Elle était relativement élevée $(6 \%)$ pour ce qui est attendu des interventions de classe 1 d'Altemeier (chirurgie propre). En effet, ces interventions qui ont constitué l'essentiel des procédures au cours de la mission sont affectées d'un risque infectieux de 1 à $2 \%$ (Waddel 1994). Nos résultats doivent être interprétés en tenant compte des conditions de travail qui sont rarement optimales en chirurgie foraine. Ces circonstances créent à chaque geste opératoire les conditions d'une chirurgie propre contaminée ; ce qui justifie l'intérêt d'une antibioprophylaxie systématique. La morbidité a été source de ré-intervention chez deux patients, rappelant l'intérêt d'établir des procédures de surveillance 
clinique et une permanence de soins qui doit couvrir au moins la période opératoire précoce.

\section{Conclusion}

Les affections les plus fréquentes en milieu rural peuvent être traitées efficacement dans le cadre d'une mission chirurgicale foraine. Cette approche, qui doit tenir compte de conditions de travail particulières, est une solution pour faciliter l'accès aux soins chirurgicaux dans les zones où le plateau technique humain et matériel est limité.

\section{References:}

1. Kushner, A.L., Cherian, M. N., Noel, L., Spiegel, D. A., Groth, S.,\&Etienne, C. (2010). Addressing the Millennium Development Goals from a surgical perspective: essential surgery and anesthesia in 8 low- and middle-income countries.Arch Surg.;145(2):154-9.

2. Thomas, G., Richards, F.O. Jr., \&Eigege, A.(2009). A pilot program of mass surgery weeks for treatment of hydrocele due to lymphatic filariasis in central Nigeria. Am J Trop Med Hyg; 80:447-451.

3. Aderounmu, A.O., Afolayan, S.A., Nasiru, T.A., Olaore, J.A., Adeoti, M. L.,\& Adelasoye, M.(2008). Rotational rural surgery for the poor in developing countries. Trop Doct; 38:141-4.

4. Ginzburg, E., O'Neill, W.W., Goldschmidt-Clermont, P.J., de Marchena, E., Pust, D., \&Green, B.A. (2010). Rapid medical relief-Project Medishare and the Haitian earthquake. $N$ Engl J Med.11;362(10).

5. Harouna, Y. (2000). Le vécu de la pathologie herniaire par le chirurgien africain : 1'exemple du Niger (Afrique). Med Afr Noire ; 47 (7).

6. Programme Mondial pour l'Elimination de la Filariose Lymphatique. Rapport d'une consultation informelle sur les méthodes chirurgicales pour traiter les manifestations urogénitales de la filariose lymphatique WHO /CDS /CEE/2002 .33.

7. Pelissier $\mathbf{E}$.Traitement chirurgical des hernies inguinales par voie inguinale. Encycl Med Chir, Techniques chirurgicales -Appareil digestif, 40-110, 2000, 11p.

8. Pallas, G., Simon,F., Sockeel, P., Chapuis, O.,\&Jacovici R. (2000).Hernie inguinale en Afrique et coelioscopie : utopie ou réalisme? Med Trop ; 60 :389-394. 
9. Koumare AK, Diop AK, Ongoiba N, Bouare M, etal. Evaluation rétrospective de 4539 cures de hernies inguinales effectuées par des médecins généralistes de districts au Mali. Med Afr Noire 1991; $38: 137-141$.

10. Pelissier E, Marre P, Damas JM. Traitement des hernies inguinales choix d'un procédé Encycl Med Chir, Techniques ChirurgicalesAppareil digestif, 40-138, 2000,4p.

11. Hay, J.M., Boudet, M.J., Fingerhut, A., Pourcher, J., Hennet, H., Habib, E., ... Flamant, Y .(1995). Shouldice Inguinal Hernia Repair in the male adult: The Gold Standard? A Multicenter Controlled Trial in 1578 Patients. Ann Surg; 222:719-727.

12. Sanders,DL.,Porter, C.S., Mitchell, K.C.,\&Kingsnorth, A.N. (2008). A prospective cohort study comparing the African and European hernia. Hernia; 12:527-9.

13. Waddel,T.K.,\&Rotstein, O.D.(1994). Antimicrobial prophylaxis in surgery. Committee on Antimicrobial Agents. Canadian Infectious Disease Society. CMAJ 151:925-31. 